\title{
PRESIÓN DE CAZA DE LA COMUNIDAD NATIVA MUSHUCKLLACTA DE CHIPAOTA, ZONA DE AMORTIGUAMIENTO DEL PARQUE NACIONAL CORDILLERA AZUL, PERÚ
}

\section{HUNTING PRESSURE IN THE MUSHUCKLLACTA DE CHIPAOTA NATIVE COMMUNITY, BUFFER ZONE OF THE CORDILLERA AZUL NATIONAL PARK}

\author{
Adrián Sánchez ${ }^{1,2}$ y Pedro Vásquez ${ }^{3}$
}

\section{Resumen}

Se determinó la presión de caza para el año 2005 en la comunidad nativa Mushuckllacta de Chipaota, ubicada en la zona de amortiguamiento del Parque Nacional Cordillera Azul - Perú. La metodología empleada se basó en el registro de los animales cazados y censos en tres regiones distintas, empleándose las pruebas estadísticas nMDS y ANOSIM para su diferenciación. Como resultado se encontraron diferencias significativas entre las regiones; indicando alteraciones en la estructura de la comunidad de fauna silvestre de acuerdo a su cercanía a los principales centros poblados. Adicionalmente se estimó la densidad global para 13 especies de animales. A partir de las densidades obtenidas en la "Región Sur" (identificada como de ligera intervención humana) y considerando los valores de la literatura (Robinson \& Redford, 1991), se generó un Modelo de Sostenibilidad, en base al cual se infiere que la caza de Mazama americana, Agouti paca, Eira barbara y Leopardus pardalis no es sostenible. Se muestra que 15 especies se encuentran bajo procesos de extinción local; y que probablemente Ateles sp., Psophia leucoptera, Pipile cumanenses y Aburria aburri han sido extirpadas del área de uso comunal.

Palabras clave: Presión de caza, Fauna silvestre, Sostenibilidad, Extinción local, Parque Nacional Cordillera Azul

\begin{abstract}
This study determined the hunting pressure for the year 2005, of the Mushuckllacta de Chipaota native community, located in the buffer zone of the Cordillera Azul National Park Peru. Hunted animals were registered, and censuses were made in three different regions of the study area, using nMDS and ANOSIM statistical tests to assess differences between regions. Significant differences where found; suggesting disturbances in the wild fauna community related to their closeness to the main populated centers. Global densities were estimated for 13 species. Taking densities from the "south-region" (identified as one with lowest human intervention) and also considering values in the literature (Robinson \& Redford, 1991) a Sustainable Model was generated which indicated that the hunting of Mazama americana, Agouti paca, Eira barbara and Leopardus pardalis was not sustainable. It is also showed that 15 species are under local extinction processes; and that it is quite probable that Ateles sp., Psophia leucoptera, Pipile cumanenses and Aburria aburri had been extirpated from the area used by the community.

Key words: Hunting pressure, wild animals, Sustainability, Local extinction, Cordillera Azul National Park
\end{abstract}

\section{Introducción}

La caza es un factor determinante cuando se habla de la conservación de muchas especies; ya que es una de las principales causas de la pérdida de fauna silvestre, debido a que se hace, en muchos casos, de manera insostenible (Robinson \& Bodmer, 1999). En Bodmer et al. (1997b) se señala que, a lo largo de la mayor parte del bosque tropical, la caza no manejada es muy común, teniendo como consecuencia la disminución de las poblaciones animales, a menudo a niveles tan bajos que las extinciones locales se harían frecuentes. Es por ello que se debe buscar un adecuado manejo de estos recursos, lo cual sería posible si se contara con suficiente información biológica de los animales de caza e información socioeconómica de aquellos que usan la fauna.

El problema que da origen a esta investigación, es justamente la tendencia de la fauna silvestre a disminuir en riqueza y abundancia dentro de los límites del área de uso de la Comunidad Nativa Mushuckllacta de Chipaota $(\mathrm{CNMCH})$. Frente a este problema y con la finalidad de orientar un manejo adecuado del recurso fauna, teniendo en consideración la presión de caza y la construcción de conclusiones sobre su sostenibilidad, se buscó: determinar la oferta de la fauna silvestre disponible para la caza; contar con un registro de animales cazados; y, comparar la 
oferta y el aprovechamiento de los animales para determinar la presión de la cacería.

Es importante mencionar que esta investigación se realizó dentro del Programa de Investigación de la ONG CIMA-Cordillera Azul, con el apoyo de la $\mathrm{CNMCH}$ y de la Jefatura del Parque Nacional Cordillera Azul, y viene a ser la continuación de un estudio realizado entre el mes de Diciembre del 2004 y Abril del 2005 (Klebelsberg, 2005).

A través de esta investigación (desarrollada entre Septiembre y Noviembre del 2005) y tomando en cuenta los resultados de Klebelsberg (2005), se pretende dar un alcance de la capacidad que tienen las poblaciones animales de las distintas especies del bosque para soportar el impacto continuo de la caza en la comunidad.

\section{Materiales y métodos}

Área de estudio: La Comunidad Nativa Mushuckllacta de Chipaota, fundada en 1946 y ubicada dentro de la zona de amortiguamiento (extremo noroeste) del Parque Nacional Cordillera Azul (PNCA) (Figura 1), se encuentra en el distrito de Chazuta, provincia y departamento de San Martín; teniendo como única vía de acceso el transporte fluvial, cruzando el río Huallaga desde el pueblo de Chazuta hasta el puerto de la comunidad.

El área titulada tiene una extensión de 6146.49 ha, mientras que el área de uso comunal comprende aproximadamente 13594.95 ha. Dentro del área de uso se encuentran dos sectores de importante agregación poblacional: "Mushuckllacta de Chipaota" y "Santa Rosa de Chipaota Viejo" (Figura 1). Siendo el primero el principal y en donde se encuentran el puerto, la posta médica, y un colegio de educación primaria para la comunidad que alberga aproximadamente a 350 familias. En el Plan Maestro del PNCA se ha considerado a esta zona como una de las "áreas críticas" (INRENA y CIMA, 2004).

El área de estudio está sujeta a un patrón de períodos secos, entre los meses de Junio o Julio hasta Octubre o Noviembre. El tipo de hábitat identificado para la zona es "Bosque de Colina Alta", la zona de vida que predomina es el bosque húmedo tropical (bh$\mathrm{T}$ ), y en la zona del Huallaga, predomina el bosque seco tropical (bs-T) (INRENA y CIMA, 2004).

El área de uso fue dividida en tres regiones (Figura 1). La Región Norte, la R. Sur y la R. Central. Estas regiones fueron generadas debido al contraste en cuanto a su lejanía al puerto de la comunidad. La Región Norte está mucho más cerca al río Huallaga que la Región Sur, donde por diferentes motivos se concentra la población. En este sentido es importante resaltar que en el extremo norte hay una amplia zona deforestada.

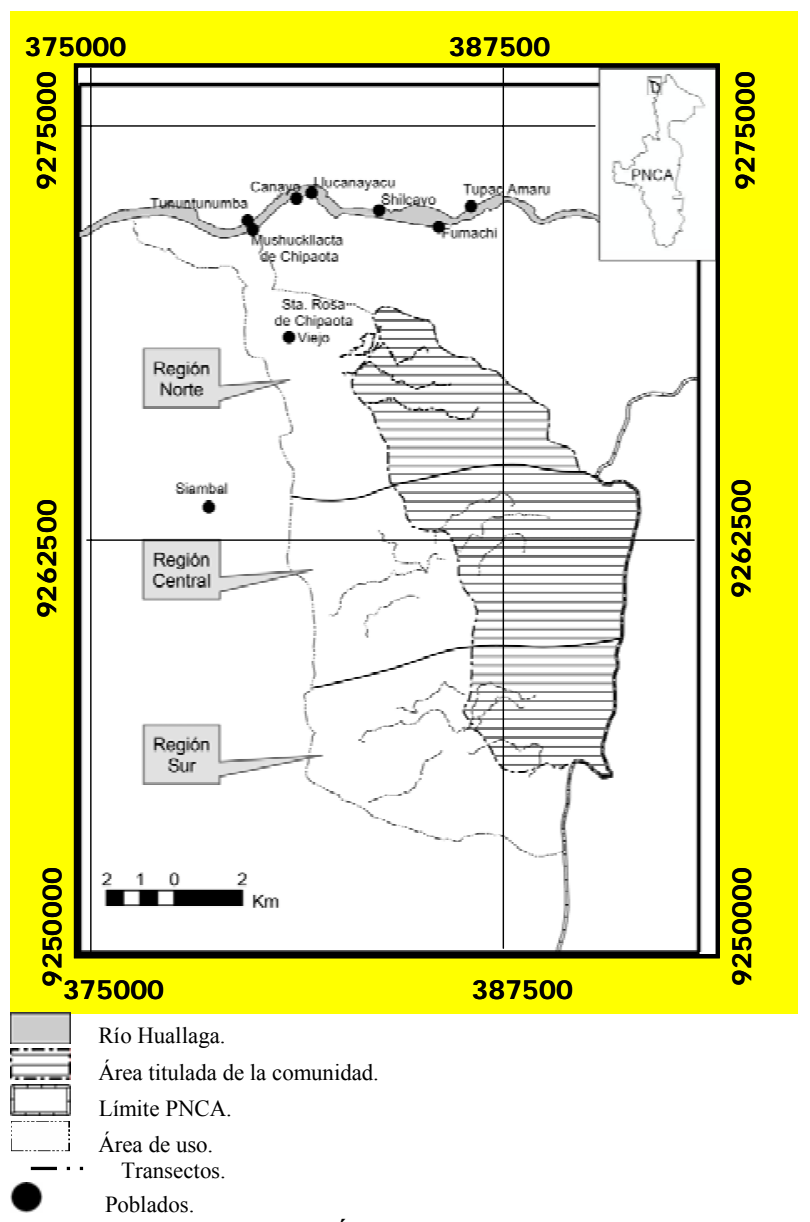

Figura 1. Área de estudio.

Muestreo:

a. Registro de animales cazados: A través de una asamblea comunal se eligió a los comuneros que participaron en el proyecto y que posteriormente fueron capacitados para la toma de datos. Se trabajó con tres comuneros que realizaron informes diarios a lo largo de toda el área de uso.

b. Censos: Se usó la metodología Distance Sampling (Buckland et al., 2001) a través de transectos para la estimación de densidades. Cada equipo de censo estuvo conformado por un observador (miembro de la $\mathrm{CNMCH}$ o guardaparque) y un encargado del equipo responsable del proyecto. El trabajo se iniciaba diariamente alrededor de las 6:00 de la mañana y los transectos se caminaron a una velocidad aproximada de $1.25 \mathrm{~km} / \mathrm{h}$, como recomienda Peres (1999). Para animales gregarios, el grupo constituyó la unidad muestral y se utilizó al primer individuo visto para el cálculo de las distancias. Se anotaron también registros indirectos de la presencia de los animales en estudio. No se realizaron censos mientras llovía y si las lluvias persistían por más de 10 minutos el censo era cancelado. 
Análisis de datos:

a. Análisis multivariado de la abundancia de fauna silvestre.

Se usó el paquete estadístico R v.1.9.1. Se clasificó la totalidad de transectos evaluando las abundancias de avistamientos relativos al esfuerzo (Km. recorridos) por medio del análisis multivariado Escalamiento Multidimensional no Métrico (nMDS, por sus siglas en inglés). Para el análisis se empleó el índice de Sorensen aplicado a datos cuantitativos (McCune \& Grace, 2002), haciendo una transformación de raíz cuadrada sobre los datos para reducir la distorción causada por especies muy abundantes. $\mathrm{La}$ significancia de las diferencias entre regiones se evaluó con la prueba no paramétrica Análisis de Similaridad (ANOSIM, por sus siglas en inglés).

b. Cálculo de la densidad poblacional.

Se usó el paquete DISTANCE v.4.1, recomendado para censos de fauna silvestre neotropical (Harris \& Burnham, 2002; Wilson et al., 1996; Bodmer et al., 1997; Peres, 1999). Se estimó la densidad global y la densidad de la Región Sur; esta última para el posterior análisis de sostenibilidad.

c. Estimación de la sostenibilidad de caza.

Se empleó el Modelo de Producción (Robinson \& Redford, 1991; Robinson \& Bodmer, 1999). El enfoque del modelo permite evaluar si la cosecha actual es no sostenible en el tiempo, calculando una producción máxima posible, disponible para cazadores, $P$.

Robinson \& Redford (1991) proponen que los cazadores pueden extraer el $60 \%$ de la producción, para animales cuya última reproducción ocurre a una edad menor a 5 años, $40 \%$ de la producción, para animales cuya edad de la última reproducción está entre 5 y 10 años, y $20 \%$ de la producción, para animales cuya última reproducción ocurre con más de 10 años. Este factor de extracción puede ser llamado $f_{e}$.

Así, se compara el número de animales cazados, $C$, con $P . P$ es un valor disponible en la literatura (Robinson \& Redford, 1991) calculado a partir de la tasa de crecimiento poblacional $\lambda_{\max }$ también en la literatura (Bodmer et al., 1997 ; Robinson \& Redford, 1991), y de la densidad de las especies en áreas poco o no perturbadas por el hombre $(D)$. En el estudio, se empleó dicho $P$ de la literatura y el estimado a partir de las densidades calculadas en la Región Sur (considerada como un área poco perturbada).

Con los datos de registro de caza se calculó la captura anual por especie $(\mathrm{Ca})$, dividiendo al número de individuos capturados entre el tiempo que tomó el registro de caza y multiplicándolo por el número total de días en un año (365). El número de animales cazados por $\mathrm{km}^{2}$ para el año $2005, C$, se calculó como sigue:

$$
C=C a / 116.97
$$

El valor $116.97 \mathrm{~km}^{2}$ corresponde a la extensión del área de caza, y se asume que el registro contempla a todos los animales cazados dentro del área de estudio.

\section{Resultados y discusión}

Esfuerzo de muestreo:

Censos Región Norte.- Cuatro transectos de 3 a 4 km. Recorridos entre 14 a 15 veces cada uno. Esfuerzo total, $207.4 \mathrm{~km}$.

Censos Región Central.- Seis transectos de 2.5 a 4 km. Recorridos entre 4 a 7 veces cada uno. Esfuerzo total, $116.9 \mathrm{~km}$.

Censos Región Sur.- Seis transectos de 3 a $4 \mathrm{~km}$. Recorridos entre 10 y 12 veces cada uno. Esfuerzo total, $246.425 \mathrm{~km}$.

Resgistro de caza.- Un comunero en cada región. 188 días de colecta de datos.

Registro de fauna:

Para el año 2005 se consiguió el registro de las especies listadas en la Tabla 1, que incluye a todas las especies registradas tanto visualmente como indirectamente, y también a especies registradas como animales cazados.

Se enlistaron 37 especies identificadas y 10 no identificadas. Teniendo un total de por lo menos 47 especies de importancia para los moradores.

Considerando el número de especies o taxones registrados en cada lugar existe un gradiente entre regiones. En la Figura 2 se puede ver que a medida que hay un alejamiento del río Huallaga, la riqueza de especies aumenta. El número de especies o taxas identificados en la Región Sur para el 2005, iguala a la riqueza encontrada dentro del PNCA (evaluación realizada en la época lluviosa de este año por Klebelsberg, 2005), aunque se presume que el registro podría ser mucho mayor para este lugar ya que en el PNCA solamente se levantaron datos en la época lluviosa.

Durante la evaluación de Klebelsberg (2005) se realizaron entrevistas a los moradores de mayor edad, donde se les preguntó sobre los animales que veían anteriormente y que ya no se encuentran en el lugar. Así, se generó un listado de catorce especies posiblemente extirpadas de la R. Norte y dos de la R. Sur. La Tabla 2 nos muestra que sí había una riqueza de especies semejante entre regiones y que, el número ha ido disminuyendo en distinta magnitud según el lugar. El presente estudio, al incrementar el esfuerzo de muestreo, registró la presencia de Tapirus terrestris en la R. Norte como animal cazado y en la R. Sur mediante el registro de huellas. 
Diciembre 2007

Tabla 1. Lista de especies registradas. Registros directos (avistamientos, vocalizaciones y animales cazados) e indirectos (huella, comedero, olor, madriguera).

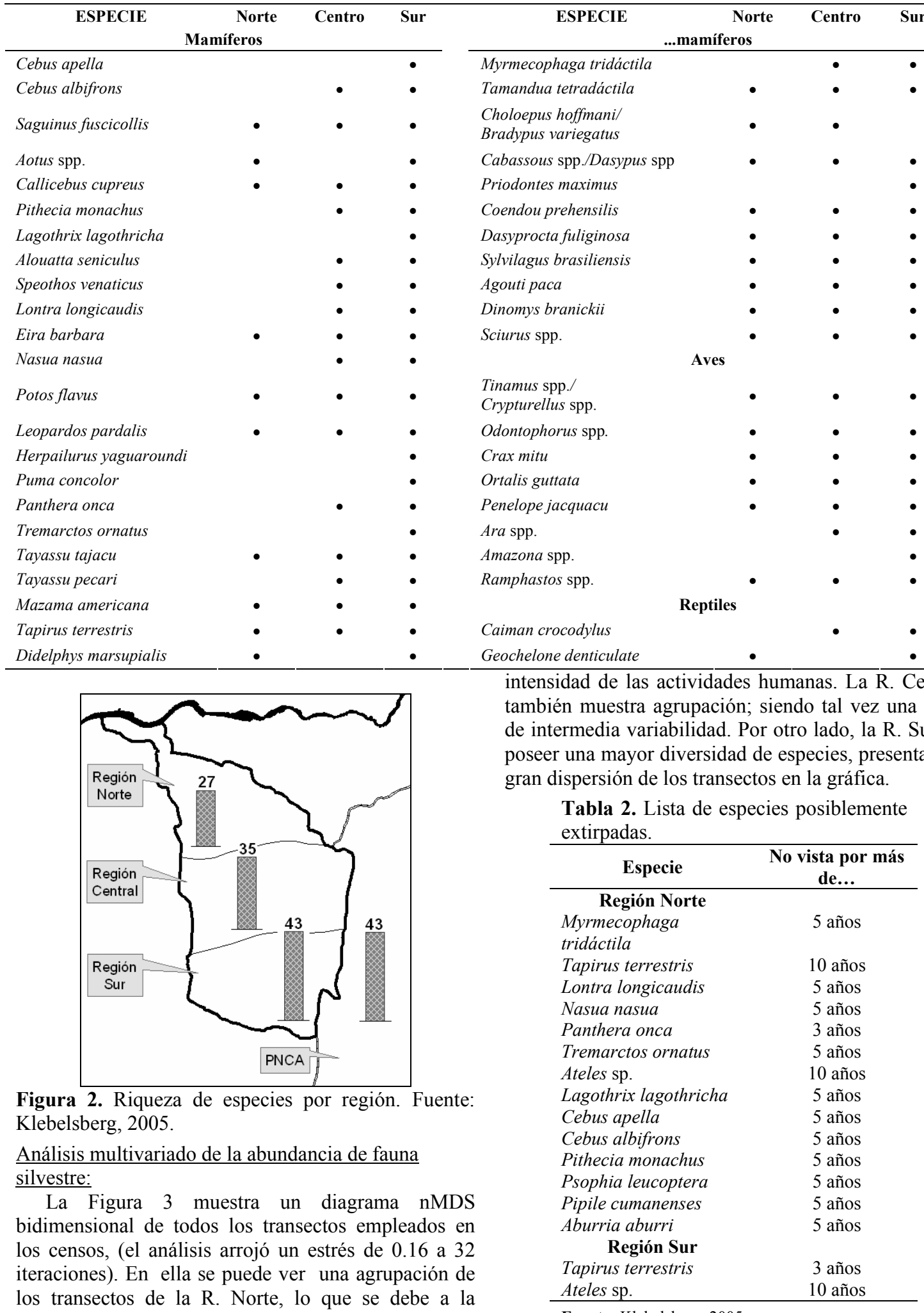
reducida riqueza de especies de la zona, producto de la

Fuente: Klebelsberg, 2005. 
El Análisis de Similaridad (ANOSIM) para las tres regiones, generó un valor de $\mathrm{R}$ de 0.4 ( $\mathrm{p}=0.001$ basado en 1000 permutaciones), mostrando diferencias significativas de por lo menos una de las regiones considerando las abundancias relativas.

Un análisis ANOSIM, únicamente comparando las regiones Norte y Sur nos arroja un $\mathrm{R}$ de 0.401 y un $\mathrm{p}=$ 0.024. Cuando se comparan las regiones Norte $\mathrm{y}$ Central se obtiene un $\mathrm{R}=0.421$ y un $\mathrm{p}=0.013$. El resultado es igualmente significativo al evaluar las regiones Central y $\operatorname{Sur}(\mathrm{R}=0.383$ y $\mathrm{p}=0.002)$. Todas estas pruebas basadas en 1000 permutaciones. Se puede decir entonces que existe evidencia estadística a un nivel de significación de 0.05 para afirmar que las regiones del área en estudio son diferentes entre sí con respecto a la abundancia de fauna silvestre de caza.

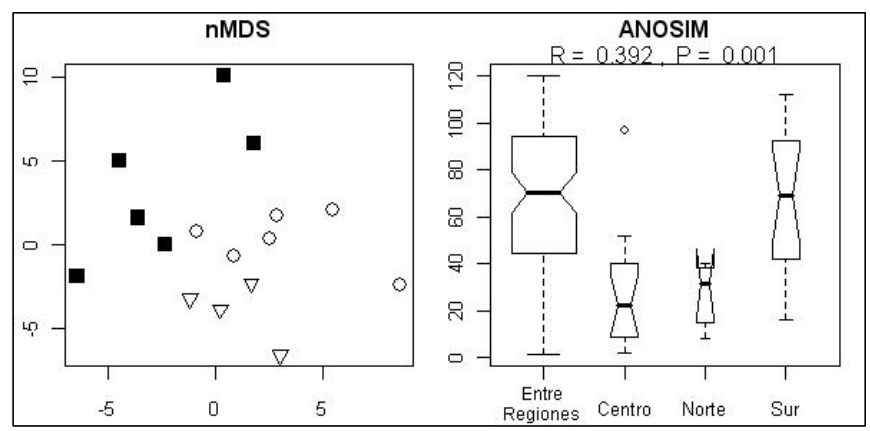

Figura 3. nMDS y ANOSIM - Registros por transecto. nMDS: izquierda. $\nabla$ Región Norte. $\circ$ Región Central.

- Región Sur.

ANOSIM: derecha.

Densidad de las poblaciones de fauna silvestre:

Se estimaron las densidades de la R. Sur (Tabla 3) y las densidades globales (Tabla 4). Según conversaciones con los comuneros, la aparición de Tayassu pecari es esporádica y en grupos de alrededor de 300 individuos; habiéndose registrado el único avistamiento de aproximadamente 250. Para el cálculo de su densidad se usó el área no deforestada (116.97 $\mathrm{km}^{2}$ ) y el único registro como el total de individuos en el área.

Registro de especies cazadas:

Se registraron 103 cazadores, encontrándose diferencias significativas entre regiones $\left(X^{2}, \mathrm{p}=\right.$ 4.682e-06). La división porcentual entre regiones se muestra en la Figura 4. El registro arrojó un total de, por lo menos, 1373 animales cazados. No se encontraron diferencias significativas entre regiones $\left(X^{2}, \mathrm{p}=0.2304\right)$ (Figura 4).

No se registraron movimientos importantes de cazadores entre regiones. La caza se da principalmente en zonas aledañas a sus viviendas y dentro de los territorios comunales, encontrándose un pequeño porcentaje de cacería fuera del área de uso (2.64\%). Se registraron mayores números de animales cazados en las regiones Norte y Central. Aunque no hubieron diferencias significativas, se puede pensar que éstas se irán acentuando ya que la mayor presencia humana en la R. Norte ya no permite que se encuentren tantos animales, y la cercanía a puertos comerciales facilita la inserción de los pobladores en actividades económicas más diversas. La relación entre las diferencias encontradas con respecto al número de cazadores, y las no encontradas del número de animales cazados muestra que esta diversificación actualmente existe.

Tabla 3. Densidades estimadas para la Región Sur, 2005. CV: Coef. de variación. G: Tamaño de grupo. D: Distance. Hn: Modelo Half-normal. Hnc: Modelo Half-normal, con ajuste de cosenos. $\mathrm{H}$ : Se asume el único registro como la totalidad de Tayassu pecari en el área.

\begin{tabular}{|c|c|c|c|}
\hline & \multicolumn{3}{|c|}{$\begin{array}{c}\text { Región Sur } 2005 \\
\text { Región de ligera intensidad de } \\
\text { caza }\end{array}$} \\
\hline & Ind $/ \mathbf{k m}^{2}$ & $\% \mathrm{CV}$ & Método \\
\hline Tayassu tajacu & 4.386 & 49.89 & D Hnc \\
\hline Tayassu pecari & 2.137 & - & $\mathrm{H}$ \\
\hline Mazama americana & 2.267 & 33.70 & D Hnc \\
\hline Pithecia monachus & 5.692 & 48.40 & D Hn \\
\hline Callicebus cupreus & 4.050 & 46.20 & D Hn \\
\hline Saguinus fuscicollis & 42.980 & 32.30 & D Hnc \\
\hline $\begin{array}{l}\text { Dasyprocta } \\
\text { fuliginosa }\end{array}$ & 13.380 & 16.65 & D Hn \\
\hline Sciurus spp. & 48.331 & 27.66 & D Hnc \\
\hline Odontophorus spp. & 11.272 & 28.65 & D Hn \\
\hline Tinamidae & 24.208 & 17.91 & D Hnc \\
\hline Crax mitu & 2.669 & 29.91 & D Hn \\
\hline Penelope jacquacu & 30.684 & 15.69 & D Hnc \\
\hline Ortalis guttata & 3.347 & 44.84 & D Hn \\
\hline
\end{tabular}

Tabla 4. Densidades globales. CV: Coef. de variación. D: Distance. Hn: Modelo Half-normal. Hnc: Modelo Half-normal, con ajuste de cosenos. $\mathrm{H}$ : Se asume el único registro como la totalidad de Tayassu pecari en el área.

\begin{tabular}{|c|c|c|c|}
\hline & \multicolumn{3}{|c|}{$\begin{array}{c}\text { Densidad global } \\
2005\end{array}$} \\
\hline & Ind $/ \mathbf{k m}^{2}$ & $\% \mathrm{CV}$ & Método \\
\hline Tayassu tajacu & 9.114 & 36.48 & D Hnc \\
\hline Tayassu pecari & 2.137 & - & $\mathrm{H}$ \\
\hline $\begin{array}{l}\text { Mazama } \\
\text { americana }\end{array}$ & 2.115 & 33.06 & D Hnc \\
\hline Pithecia monachus & 2.455 & 48.42 & D Hn \\
\hline Callicebus cupreus & 4.246 & 36.74 & D Hn \\
\hline $\begin{array}{l}\text { Saguinus } \\
\text { fuscicollis }\end{array}$ & 36.986 & 24.13 & D Hnc \\
\hline $\begin{array}{l}\text { Dasyprocta } \\
\text { fuliginosa }\end{array}$ & 15.429 & 8.33 & D Hnc \\
\hline Sciurus spp. & 36.447 & 13.68 & D Hnc \\
\hline Odontophorus spp. & 13.514 & 26.37 & D Hnc \\
\hline $\begin{array}{l}\text { Tinamidae } \\
\text { Crax mitu }\end{array}$ & $\begin{array}{l}20.491 \\
5.546\end{array}$ & $\begin{array}{l}12.41 \\
25.27\end{array}$ & $\begin{array}{l}\text { D Hnc } \\
\text { D Hnc }\end{array}$ \\
\hline Penelope jacquacu & 12.747 & 13.20 & D Hnc \\
\hline Ortalis guttata & 5.468 & 28.46 & D Hnc \\
\hline
\end{tabular}




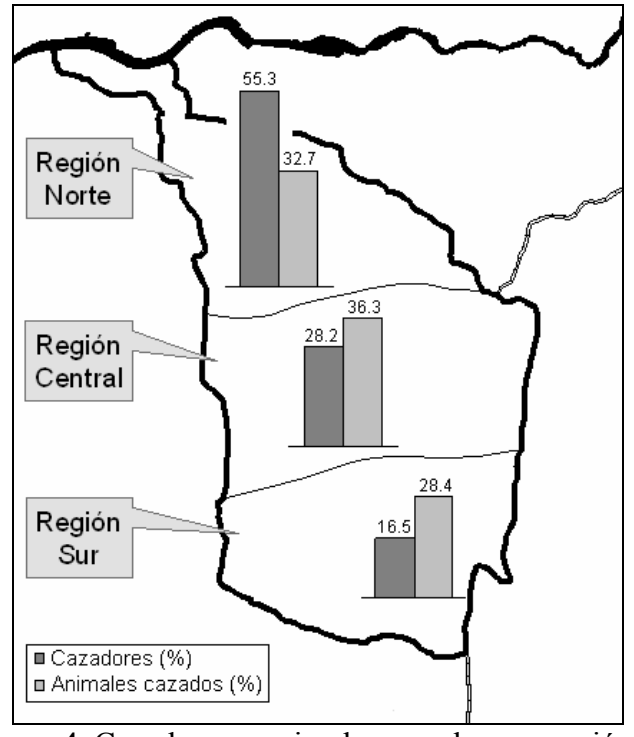

Figura 4. Cazadores y animales cazados por región.
La caza se realizó en tres modalidades: la más importante es la caza con escopeta $(68.3 \%$ de los animales cazados), la segunda modalidad es la caza con armadillera, trampa de arma de fuego (25.4\%), y la tercera es la caza con perros $(6.3 \%)$.

Los mamíferos con mayor intensidad de caza son: Dasyprocta fuliginosa, Agouti paca, Tayassu tajacu, Cabassous spp./Dasypus spp. y Mazama americana y en aves es Crax mitu (Tabla 5).

Modelo de Análisis de Sostenibilidad:

En la Tabla 6 se presentan dos alcances con los que se busca inferir sobre la sostenibilidad de la cosecha. Se exponen los valores de la Máxima Extracción Permisible $(P)$ en dos columnas. La primera, $P_{l}$, indica la máxima extracción posible calculada a partir de los datos obtenidos en este proyecto; y la segunda, $P_{2}$, muestra las estimaciones del trabajo realizado por Robinson \& Redford (1991). Ambas columnas concuerdan sobre la sostenibilidad de caza de las especies.

Con respecto a la confiabilidad del modelo, se puede considerar los valores máximos y mínimos de $P_{l}$, pudiendo analizar el caso de cinco especies. Para el caso de Tayassu tajacu, Pithecia monachus y Callicebus cupreus los valores del registro de caza se encontraron dentro del intervalo de confianza, por lo que no se puede afirmar sobre su sostenibilidad. Sin embargo, con respecto a Mazama americana, las estadísticas sí nos permiten confirmar la insostenibilidad de la caza. Finalmente, sobre la caza de Dasyprocta fuliginosa se podría pensar que se está dando en niveles sostenibles.

Considerando los valores de $P_{2}$, se infiere sobre la no sostenibilidad de caza de Mazama americana, Eira barbara, Leopardus pardalis y Agouti paca. Es importante recalcar que teniendo en cuenta las características particulares del hábitat del área de estudio, se toman estos resultados como referenciales.

Para el caso de especies como Tapirus terrestres, Pithecia monachus, Alouatta seniculus, Cebus albifrons y Cebus apella, el modelo generado (únicamente a partir de valores de la literatura) no permite inferir sobre la no sostenibilidad de caza. Sin embargo, justamente para estos animales es que se aprecia un repliegue hacia zonas de menor 
Lo mismo ocurre para especies como Lagothrix lagothricha, Panthera onca, Tremarctos ornatus, Myrmecophaga tridactyla, Lontra longicaudis, Speothos venaticus, Nasua nasua, Priodontes maximus, Puma concolor y Herpailurus yaguaroundi; cuya presencia es casi nula dentro de la comunidad, habiéndose registrado cada especie de manera indirecta o como registro de caza a lo largo de todo el año y sólo en las regiones Central y Sur.

Siendo el registro de estas especies restringido a las regiones Central y Sur, y su aparición en la $\mathrm{R}$. Norte -como es el caso de Tapirus terrestrisesporádica, es que se comprueba que la caza y las demás actividades humanas ya han producido efectos importantes en la reducción de sus poblaciones, por lo que se puede pensar que están bajo un proceso de extinción local.

Ateles sp., Psophia leucoptera, Pipile cumanenses, Aburria aburri se enlistan en la Tabla 2 como especies no vistas por más de 5 años y no han arrojado registros de presencia durante este proyecto. Es muy probable que éstas sean ya especies extirpadas.

Sobre el resto de especies enlistadas en la Tabla 1, y que no se mencionan en esta sección; se considera que se necesita mayor información para poder concluir verazmente sobre la situación de sus poblaciones. Información concerniente a su abundancia o, en el caso de las aves de porte grande, sobre la dinámica de sus poblaciones ya que, por ejemplo, no se cuenta con datos de Máxima Extracción Permisible en la literatura.

\section{Conclusiones}

La caza es muy importante para los moradores de la comunidad, en donde existen por lo menos 47 especies de importancia de caza. El Modelo de Análisis de Sostenibilidad (considerando valores de la literatura), nos permitió alertar sobre la no sostenibilidad de la caza de Mazama americana, Eira barbara, Leopardus pardalis y Agouti paca. Considerando las densidades calculadas en el área de estudio se pudo advertir únicamente sobre la no sostenibilidad de caza de Mazama americana.

La estructura de la comunidad de fauna silvestre está siendo alterada, llevándose a cabo un proceso de extinción local. Urge tomar medidas para asegurar la conservación de: Mazama americana, Eira barbara, Leopardus pardalis, Agouti paca, Tapirus terrestres, Pithecia monachus, Alouatta seniculus, Cebus albifrons y Cebus apella que aún forman parte importante del ecosistema; y Lagothrix lagothricha, Panthera onca, Tremarctos ornatos, Myrmecophaga tridactyla, Lontra longicaudis, Speothos venaticus, Nasua nasua, Priodontes maximus, Puma concolor y Herpailurus yaguaroundi que se encuentran esporádicamente en la zona. Se considera que las especies: Ateles sp., Psophia leucoptera, Pipile cumanenses y Aburria aburri muy probablemente se encuentran extirpadas del área de estudio. Habiéndose mostrado que Agouti paca es una de las especies bajo mayor presión de caza, es imperativo realizar estudios sobre la abundancia de esta especie en el lugar.

Es necesario difundir información relacionada a los efectos de la presión de caza en la comunidad y promover el desarrollo de un programa de conservación de los animales usualmente cazados en la zona con normas de uso de recursos tomadas por los propios comuneros. Así como un plan de monitoreo desde el Parque Nacional Cordillera Azul que permita hacer el seguimiento del estado de las poblaciones de fauna en las zonas cercanas a la zona protegida, manteniendo un registro continuo de cacería. Asimismo debe realizarse un estudio de caracterización del bosque para evaluar el impacto que está sufriendo el hábitat.

En evaluaciones posteriores, es recomendable aumentar el esfuerzo de muestreo en los censos; especialmente para los casos de Tayassu tajacu y Mazama americana, especies muy apreciadas por su carne y tamaño y que, en esta investigación, muestran un alto $\mathrm{CV}$ en el cálculo de las densidades.

\section{Agradecimientos}

Este trabajo se realizó con el apoyo y financiamiento de la ONG CIMA (Centro de Conservación, Investigación y Manejo de Áreas Naturales); gracias especialmente a Fernando Rubio y Tatiana Pequeño por sus aportes desde la formulación de la investigación. A la Comunidad Nativa Mushuckllacta de Chipaota, a cada una de las personas que participaron en el proyecto y a todos sus moradores. A la Jefatura del Parque Nacional Cordillera Azul - INRENA. A Eva Klebelsberg, cuyo estudio ha sido parte fundamental de este proyecto. A los miembros voluntarios del proyecto. A quienes no dudaron en brindar apoyo para sacar adelante este trabajo, Claudia Caro y Jean Paul Perret.

\section{Literatura citada}

Aquino R. \& Calle A. 2003. Evaluación del estado de conservación de los mamíferos de caza: un modelo comparativo en comunidades de la Reserva Nacional Pacaya Samiria (Loreto, Perú). Revista peruana de biología,. 10(2): 163-174.

Bodmer R.E., Aquino R., Puertas P.E., Reyes C.J., Fang T.G. \& Gottdenker N.L. 1997a. Manejo y uso sustentable de pecaríes en la amazonía peruana. Ocasional Paper of the UICN Special Survival Commission $\mathrm{N}^{\circ}$ 18. UICN-Sur, Quito, Ecuador y Secretaría CITES, Ginebra, Suiza. Pp.iv + 102.

Bodmer R.E., Eisenberg J.F. \& Redford K.H. 1997b. Hunting and the likelihood of extintion of amazonian mammals. Conservation Biology. 11 (2): 460-466.

Buckland S.T., Anderson D.R., Burnham K.P., Laake J.L., Borchers D.L \& Thomas L. 2001. Introduction to distance sampling. New York, Oxford University Press. 
Harris R.B. \& Burnham K.P. 2002. On estimating wildlife densities from line transect data. Acta Zoological Sinica. 48: 812-818.

Instituto Nacional de Recursos Naturales (INRENA) \& Centro de Conservación, Investigación y Manejo de Áreas Naturales (CIMA). 2004. Plan Maestro del Parque Nacional Cordillera Azul. Versión en CD. Lima, CIMA.

Klebelsberg E. 2005. Informe del trabajo de campo realizado con CIMA entre el 7 de diciembre del 2004 y el 24 de Junio del 2005. Documento interno. CIMACordillera Azul, Lima.

McCune B. \& Grace J.B. 2002. Analysis of ecological communities. Corvallis, Oregon State University.

Peres C.A. 1999. General guidelines for standarizing line transect surveys of tropical forest primates. Neotropical primates. 7(1): 11-16.
Robinson J. \& Bodmer R. 1999. Towards wildlife management in tropical forests. Journal of Wildlife Management. 63(1): 1-13.

Robinson J.G. \& Redford K.H. 1991. Sustainable harvest of neotropical forest mammals. En: Robinson J.G. \& Redford K.H. 1991. Neotropcal wildlife use and conservation. Chicago y London, The University of Chigago Press.

Wilson D.E., Cole F.R., Nichols J.D., Rudram R. \& Foster M.S. 1996. Measuring and monitoring biological diversity: standard methods for mammals. Washington y London, Smithsonian Institution Press.

Zapata Galos. 2001. Sustentabilidad de la cacería de subsistencia: El caso de cuatro comunidades Quichuas en la amazonía nororiental ecuatoriana. Mastozoología Neotropical / J. Neotrop. Mammal. 8(1): 59-66.

Tabla 6. Modelo de Análisis de Sostenibilidad.

\begin{tabular}{|c|c|c|c|c|c|c|c|c|}
\hline Nombre Científico & $\begin{array}{l}\text { Densidad } \\
\text { Calculada } \\
D_{1}\end{array}$ & $\begin{array}{l}\text { Densidad } \\
\text { Esperada } \\
D_{2}\end{array}$ & $f_{e}$ & $\lambda_{\max }$ & $P_{1}$ & $P_{2}$ & $\begin{array}{l}\text { Caza } \\
2005 \\
\left(\mathrm{Ind} / \mathrm{km}^{2}\right)\end{array}$ & $\begin{array}{l}\text { Caza } \\
\text { Sostenible } \\
\left(P_{1} / P_{2}\right)\end{array}$ \\
\hline Tayassu tajacu & 4.39 & 8.05 & $40^{\infty}$ & $3.49^{\psi}$ & 2.62 & $4.81^{\text {я }}$ & 1.41 & Sí/Sí \\
\hline Tayassu pecari & 2.14 & 5.24 & $40^{\infty}$ & $2.32^{\psi}$ & 0.68 & $1.66^{\text {я }}$ & 0.56 & Sí/Sí \\
\hline Mazama americana & 2.27 & 5.67 & $40^{\psi}$ & $1.49^{\psi}$ & 0.27 & 0.67 & 0.76 & $\mathrm{No} / \mathrm{No}$ \\
\hline Pithecia monachus & 5.69 & - & $10^{\Delta}$ & $1.13^{\partial}$ & 0.04 & & 0.05 & No/-- \\
\hline Callicebus cupreus & 4.05 & - & $20 *$ & $1.26^{\partial}$ & 0.13 & & 0.07 & Sí/-- \\
\hline Saguinus fuscicollis & 42.98 & - & $20 *$ & & & & 0.07 & $--/--$ \\
\hline Dasyprocta fuliginosa & 13.38 & 18.70 & $40^{\psi}$ & $3.00^{\psi}$ & 6.42 & 8.98 & 2.03 & Sí/Sí \\
\hline Sciurus spp. & 47.69 & 75.50 & $60^{\psi}$ & & & & 0.38 & --/-- \\
\hline Alouatta seniculus & - & 19.32 & $10^{\Delta}$ & $1.18^{\partial}$ & & $0.21^{\text {я }}$ & 0.00 & --/Sí \\
\hline Cebus albifrons & - & $9.50^{\ell}$ & $10^{\Delta}$ & $1.18^{\partial}$ & & $0.11^{\text {я }}$ & 0.05 & --/Sí \\
\hline Cebus apella & - & 9.82 & $10^{\Delta}$ & $1.15^{\partial}$ & & $0.09^{\text {я }}$ & 0.00 & --/Sí \\
\hline Nasua nasua & - & $13.2^{\ell}$ & $40^{\ell}$ & $1.25^{\ell}$ & & 0.79 & 0.12 & --/Sí \\
\hline Potos flavus & - & $15.1^{\ell}$ & $20^{\ell}$ & $1.34^{\ell}$ & & 0.62 & 0.37 & --/Sí \\
\hline Eira barbara & - & $0.9^{\ell}$ & $40^{\ell}$ & $1.32^{\ell}$ & & 0.07 & 0.17 & --/No \\
\hline Leopardus pardales & - & $0.8^{\ell}$ & $40^{\ell}$ & $1.58^{\ell}$ & & 0.11 & 0.13 & --/No \\
\hline Tapirus terrestres & - & 1.22 & $20^{\psi}$ & $1.22^{\psi}$ & & 0.03 & 0.02 & --/Sí \\
\hline Agouti paca & - & 11.49 & $20^{\psi}$ & $1.95^{\psi}$ & & 1.31 & 1.58 & $--/ \mathrm{No}$ \\
\hline
\end{tabular}

$\boldsymbol{D}_{\boldsymbol{1}}$ : Densidad calculada en el presente estudio $\left(\mathrm{Ind} / \mathrm{km}^{2}\right) ; \boldsymbol{D}_{2}$ : Densidad estimada por Robinson \& Redford (1991); $f_{e}$ : Factor de extracción; $\lambda_{\max }$ : Tasa finita máxima de incremento; $P_{1}$ : Máxima extracción permisible calculada en el presente estudio $\left(\mathrm{Ind} / \mathrm{km}^{2}\right) ; P_{2}$ : Máxima extracción permisible, estimada por Robinson \& Redford (1991).

${ }^{\infty}$ Bodmer et al. $\left(1997^{\mathrm{a}}\right) .{ }^{\psi}$ Robinson \& Redford (1991). ${ }^{\mathrm{A}}$ Estimado a partir del presente $f_{e} .{ }^{\Delta}$ Aquino \& Calle (2003). ${ }^{\circ}$ Bodmer $e t$ al. (1997b). *Klebelsberg (2005). 'Robinson \& Redford (1986; en Zapata, 2001).

\footnotetext{
${ }^{1}$ Afiliación en el momento de la investigación: Universidad Nacional Agraria La Molina, Facultad de Ciencias. Av. La Molina s/n, La Molina, Lima - Perú.

${ }^{2}$ Afiliación actual: Tambopata Macaw Project. Tambopata Research Center, Puerto Maldonado, Madre de Dios Perú. adriansanchezgonzalez@gmail.com

${ }^{3}$ Centro de Datos para la Conservación. Universidad Nacional Agraria La Molina, Facultad de Ciencias Forestales. Av La Molina s/n, La Molina, Lima - Perú. cdc@lamolina.edu.pe
} 